\title{
Analysis of a Substrate Integrated Waveguide Hybrid Ring (Rat-Race) Coupler
}

\author{
Ramin Dehdasht-Heydari, ${ }^{1}$ Keyvan Forooraghi, ${ }^{2}$ and Mohammad Naser-Moghadasi ${ }^{1}$ \\ ${ }^{1}$ Department of Electrical Engineering, Science and Research Branch, Faculty of Engineering, Islamic Azad University, \\ Tehran 14778 93855, Iran \\ ${ }^{2}$ Department of Electrical Engineering, Faculty of Engineering, Tarbiat Modares University (TMU), Tehran 14115 111, Iran
}

Correspondence should be addressed to Ramin Dehdasht-Heydari; dehdashtheydari@yahoo.com

Received 24 January 2014; Revised 4 April 2014; Accepted 4 April 2014; Published 28 April 2014

Academic Editor: Francisco Falcone

Copyright (c) 2014 Ramin Dehdasht-Heydari et al. This is an open access article distributed under the Creative Commons Attribution License, which permits unrestricted use, distribution, and reproduction in any medium, provided the original work is properly cited.

\begin{abstract}
This paper presents an efficient analysis of a substrate integrated waveguide (SIW) single-layer hybrid ring coupler (rat-race) for millimeter-wave and microwave applications. The scattered field from each circular cylinder is expanded by cylindrical eigenfunctions with unknown coefficients that have been solved by electric and magnetic tangential boundary on each metallic via. The coupler S-matrix is calculated by using mode matching that uses the cylindrical vector expansion analysis to minimize the computational time and provides more physical insight. To achieve higher bandwidth, the radiuses of the coupler under analysis have been optimized in Matlab code by invasive weed optimization (IWO) method, and the results have been verified by CST package. The return loss and the isolation are less than $-15 \mathrm{~dB}$, and $-18 \mathrm{~dB}$, respectively. The insertion loss is divided equally $-3 \pm 0.2 \mathrm{~dB}$, with $0 \pm 5$ and $180 \pm 10$ degrees in output ports over the operating frequency bandwidth and the agreement of phase differences in output ports has been examined objectively by feature selective validation (FSV) technique.
\end{abstract}

\section{Introduction}

Theconcept of substrate integrated waveguides was proposed [1] to overcome the drawbacks of conventional rectangular waveguide and has drawn much attention, for it can offer a promising solution to compact, low-cost, efficient, and hybrid integration of different kinds of conventional microstrip structures [2] in the same substrate at microwave and millimeter wave frequencies. In developing SIW base devices, many passive components, such as transitions, filters, oscillators, antennas, circulators, and couplers, have been studied in microwave and millimeter-wave systems [3-10].

The analysis of substrate integrated waveguide (SIW) structures has been carried out in many methods. For instance, the method of moments accompanied with the Floquet mode expansions [11], the finite-difference frequency domain (FDFD) method [12], the boundary integralresonant expansion (BI-RME) method [13] and the method of lines [14] have been applied to determine the propagation characteristics of the substrate integrated wave guides (SIWs).
Moreover, the design of a real device mostly depends on the finite element method and the method of moment, which can be time consuming and memory demanding for large structures.

Recently, several papers have made use of a cylindrical vector wave (CVW) expansion in order to study the simplified $2 \mathrm{D}$ case [15-17] and the full 3D case [18-24]. These mode expansions allow an efficient full-wave analysis of SIW structures with metallic and dielectric posts. Furthermore, there is a need for using efficient methods of mode matching and CVW to analyze some specific structures such as couplers. The design of the SIW rat race coupler basically has been presented in [25] with transition from microstrip to SIW ratrace arms. However, in [25], the output phases results (i.e. phase differences of 0 and 180 degrees) of the coupler were not mentioned at all. In addition, the dimensions of the coupler should be optimized to achieve the more relative bandwidth. Because the analysis of the coupler has not been examined, its optimization has not been carried out properly in this case. 


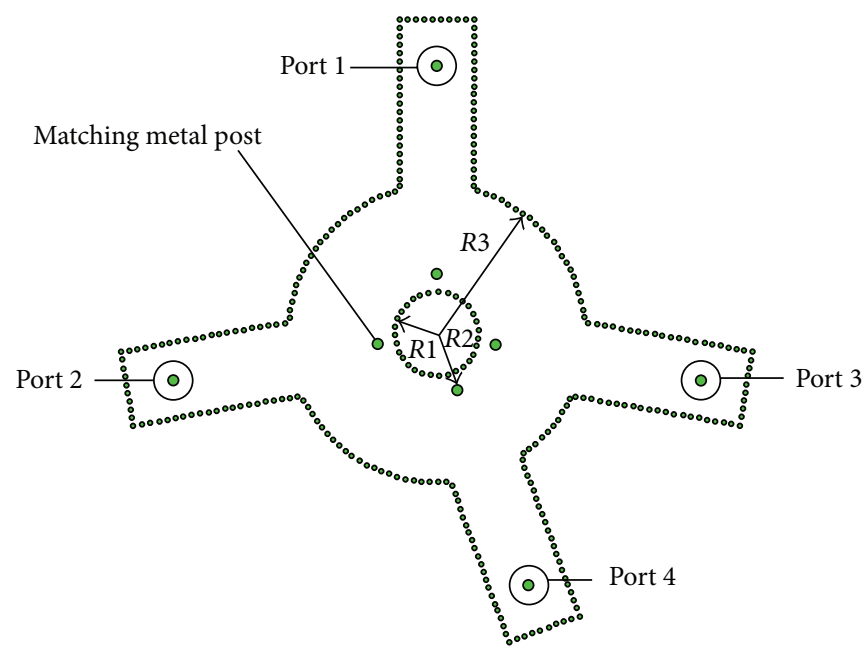

(a)

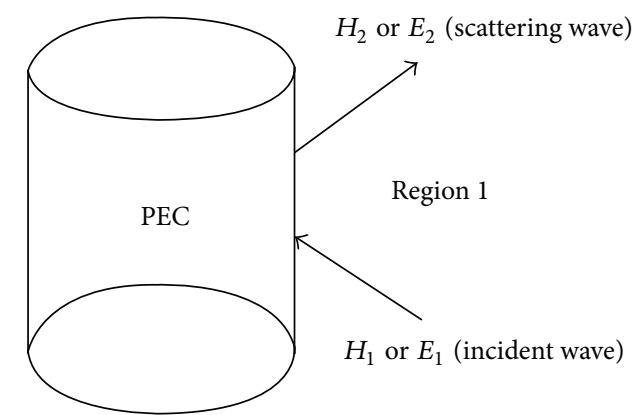

(b)

FIGURE 1: (a) Top view of the proposed rat-race coupler under analysis. (b) One via of rat-race coupler and its related waves.

In this paper, the dyadic Green's function in a cylindrical medium can be represented in terms of cylindrical vector wave eigenfunctions. So, at first we find the vector wave function for each PEC via in the SIW rat-race coupler structure which is excited by coaxial cables and shown in Figure 1. Because of using four coaxial cables instead of microstrip line transitions and a closed form of arms $(\lambda / 4$ distance from the coaxial cables), our structure in this paper is of low loss and profile.

When these vector wave expansions are known, the dyadic Green's function for our rat-race coupler can be expressed in terms of these vector wave functions. By imposing boundary conditions on each via and transferring the obtained equations to matrix, we find the unknown coefficient of CVW. In the end, we find admittance matrix from the coefficients and conveniently we can reach scattering parameters of the proposed rat-race coupler. The mode-matching method has been implemented in Matlab software to compare the results with CST package. Also, to achieve better characteristics of rat-race coupler, we use an optimization with IWO and the optimized results are compared. It can be observed that the relative bandwidth has been increased from $9 \%$ to $17 \%$ over $11.4-13.7 \mathrm{GHz}$. In addition, the agreement of the output phase results has been shown clearly with feature selective validation (FSV).

\section{Analysis of Rat-Race Coupler with Cylindrical Vector Wave (CVW) Expansion Formulations}

As the structure in Figure 1(a) is a homogeneous medium, the electric and magnetic fields fulfill the following equations:

$$
\begin{aligned}
& \nabla \times \nabla \times \mathbf{E}(\mathbf{r})-k^{2} \mathbf{E}(\mathbf{r})=0, \\
& \nabla \times \nabla \times \mathbf{H}(\mathbf{r})-k^{2} \mathbf{H}(\mathbf{r})=0,
\end{aligned}
$$

where $k$ is the wave number in dielectric substrate and $k=$ $\omega \sqrt{\mu_{0} \varepsilon_{0} \varepsilon_{r}}$.

Also, in a homogenous medium, $\mathbf{E}(\mathbf{r})$ and $\mathbf{H}(\mathbf{r})$ are derivable from scalar potential $\vartheta(r)$. Now, we can define two vector wave functions $\mathbf{P}(\mathbf{r})$ and $\mathbf{Q}(\mathbf{r})$ as the equivalence of an electric or a magnetic field. Notice that the above equation is valid for $\mathbf{Q}(\mathbf{r})$ :

$$
\nabla \cdot \mathbf{P}(\mathbf{r})=0, \quad \nabla \times \mathbf{P}(\mathbf{r}) \neq 0 .
$$

For consideration of the relation between $\vartheta(r)$ and $\mathbf{P}(\mathbf{r})$ and $\mathbf{Q}(\mathbf{r})$, let us assume the following equation for scalar potential:

$$
\left(\nabla^{2}+k^{2}\right) \vartheta(r)=0
$$

and the relation between $\vartheta(r), \mathbf{P}(\mathbf{r})$, and $\mathbf{Q}(\mathbf{r})$ is as follows:

$$
\begin{aligned}
& \mathbf{P}(\mathbf{r})=\nabla \times\left(\vartheta(r) \mathbf{a}_{n}\right), \\
& \mathbf{Q}(\mathbf{r})=\frac{1}{k} \nabla \times \mathbf{P}(\mathbf{r}),
\end{aligned}
$$

where $\mathbf{a}_{n}$ is unit normal vector on the surface.

In a cylindrical coordinate we can express in each via in region 1 (outgoing region) this harmonic scalar potential $\vartheta_{n}(r)$ :

$$
\vartheta_{n}(r)=H_{n}^{(2)}\left(k_{m} \rho\right) e^{j\left(k_{z} z-n \varphi\right)},
$$

and $H_{n}^{(2)}$ indicates the Hankel function of the second kind, $k_{m}=\sqrt{k^{2}-k_{z}^{2}}, k_{z}=m \pi / h$, and $h$ is the height of substrate in Figure 1(a). In (6), $m$ and $n$ are the modes in vertical direction ( $z$-direction) and azimuthal direction $(\varphi$ direction), respectively. Also, in this paper we consider the dependence of the SIW structure on the variation to $z$, which is expressed as an exponential term. 
By using (6) in (4) and (5) we obtain $\mathbf{P}(\mathbf{r})$ and $\mathbf{Q}(\mathbf{r})$ for Figure 1(b):

$$
\begin{aligned}
\mathbf{P}_{n}(\mathbf{r})= & \left(\frac{-1}{\rho} j n e^{j\left(k_{z} z-n \varphi\right)} H_{n}^{(2)}\left(k_{m} \rho\right)\right) \mathbf{a}_{\rho} \\
& +\left(-k_{m} \rho H_{n}^{\prime(2)}\left(k_{m} \rho\right) e^{j\left(k_{z} z-n \varphi\right)}\right) \mathbf{a}_{\varphi} \\
\mathbf{Q}_{n}(\mathbf{r})= & \left(j k_{m} k_{z} \rho H_{n}^{\prime(2)}\left(k_{m} \rho\right) e^{j\left(k_{z} z-n \varphi\right)}\right) \mathbf{a}_{\rho} \\
& +\left(\frac{1}{\rho} n k_{z} e^{j\left(k_{z} z-n \varphi\right)} H_{n}^{(2)}\left(k_{m} \rho\right)\right) \mathbf{a}_{\varphi} \\
& +\left(k _ { m } e ^ { j ( k _ { z } z - n \varphi ) } \left(-2 H_{n}^{\prime(2)}\left(k_{m} \rho\right)\right.\right. \\
& \left.\left.-\rho k_{m} H_{n}^{\prime \prime(2)}\left(k_{m} \rho\right)\right)\right) \mathbf{a}_{z},
\end{aligned}
$$

where

$$
\begin{aligned}
& H_{n}^{\prime(2)}\left(k_{m} \rho\right)=\frac{\partial}{\partial}\left(H_{n}^{(2)}\left(k_{m} \rho\right)\right), \\
& H_{n}^{\prime \prime(2)}\left(k_{m} \rho\right)=\frac{\partial}{\partial}\left(H_{n}^{\prime(2)}\left(k_{m} \rho\right)\right) .
\end{aligned}
$$

So, arbitrary electric and magnetic fields $E(r)$ and $H(r)$ in region 1 can be expressed as

$$
\begin{gathered}
H_{\text {total }}(r)=H_{1}+H_{2}, \\
E_{\text {total }}(r)=E_{1}+E_{2},
\end{gathered}
$$

where $E_{2}$ and $H_{2}$ are scattering electrical and magnetic waves from the via and $E_{1}$ and $H_{1}$ are incident waves from a magnetic source $\left(M_{s}^{\prime}\right)$ in the region 1 . To calculate $H_{1}$, we need this equation:

$$
H_{1}=-j \omega \varepsilon \iiint G_{m 2} \cdot M_{s}^{\prime} d V^{\prime},
$$

where $G_{m 2}$ is the magnetic Green function of second kind $[26,27]$ which for parallel plate waveguide by using residue theorem in cylindrical coordination explicit form is given by

$$
\begin{aligned}
G_{m 2}\left(\mathbf{r}, \mathbf{r}^{\prime}\right)= & -\frac{\hat{\rho} \hat{\rho}}{k_{0}^{2}} \delta\left(\mathbf{r}-\mathbf{r}^{\prime}\right) \\
& -\frac{1}{j 4 \pi} \sum_{m=0}^{N_{z}} \sum_{n=0}^{N_{\varphi}}\left(2-\delta_{m 0}\right) \frac{1}{k_{m}^{2} h} \\
& \times\left[P_{n}\left(k_{m}, k_{z}, \rho\right) P_{n}^{\prime}\left(k_{m}, k_{z}, \rho^{\prime}\right)\right. \\
& \left.+Q_{n}\left(k_{m}, k_{z}, \rho\right) Q_{n}^{\prime}\left(k_{m}, k_{z}, \rho^{\prime}\right)\right], \\
& \delta_{m 0}= \begin{cases}0 & \text { if } m \neq 0 \\
1 & \text { if } m=0,\end{cases}
\end{aligned}
$$

where $P_{n}$ and $Q_{n}$ for $\rho>\rho^{\prime}$ are similar as outgoing wave in region 1 for (7a) and (7b). For $\rho<\rho^{\prime}$, the equations are replaced with $\left(J_{n}\left(k_{m} \rho\right)\right)$ instead of $\left(H_{n}\left(k_{m} \rho\right)\right)$. Also, $P_{n}^{\prime}$ and $Q_{n}^{\prime}$ can be achieved by interchanging $\rho<\rho^{\prime}$ with $\rho>\rho^{\prime}$

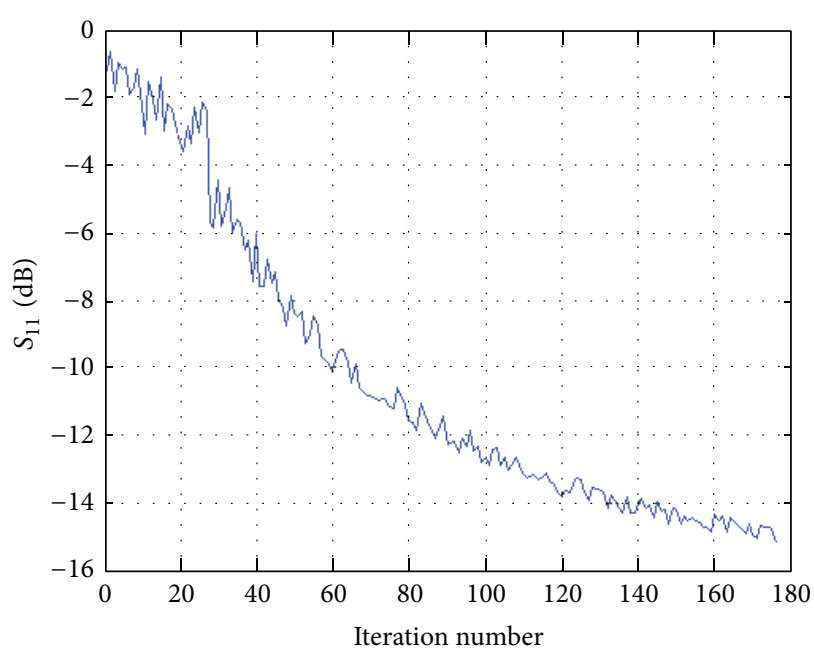

FIGURE 2: $S_{11}$ of the rat-race coupler versus IWO iteration number.

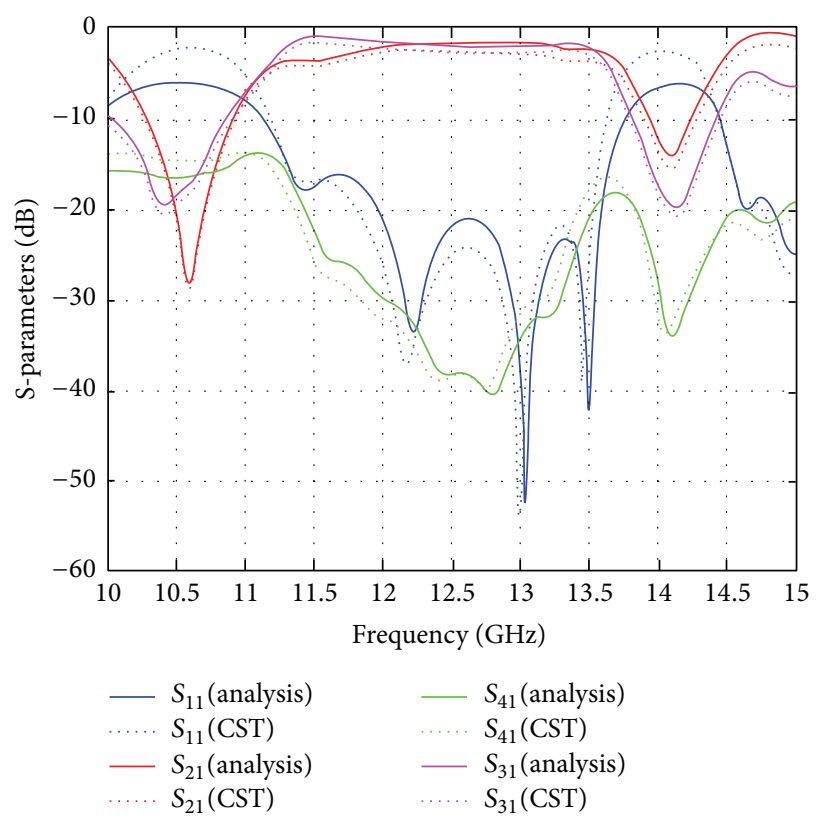

FIGURE 3: The $S$-parameters versus frequency from analysis and CST.

and vice versa. The $m$ and $n$ in (11) are eigenvalues modes in vertical and azimuthal directions, respectively.

After obtaining $H_{1}$ from (10) the electrical field $E_{1}$ is obtained from

$$
E=\frac{1}{j \omega \epsilon} \nabla \times H
$$

From (1a), (1b), and (3), we can write TE and TM modes for scattering waves $\left(\mathrm{H}_{2}, \mathrm{E}_{2}\right)$ in region 1 as $\mathrm{CVW}$ expansions:

$$
H_{2}(r)=\sum_{l=1}^{P_{\text {via }}} \sum_{m=1}^{N_{z}} \sum_{n=1}^{N_{\varphi}}\left(C_{l, m, n}^{\mathrm{TM}} P_{n}+D_{l, m, n}^{\mathrm{TE}} Q_{n}\right)
$$




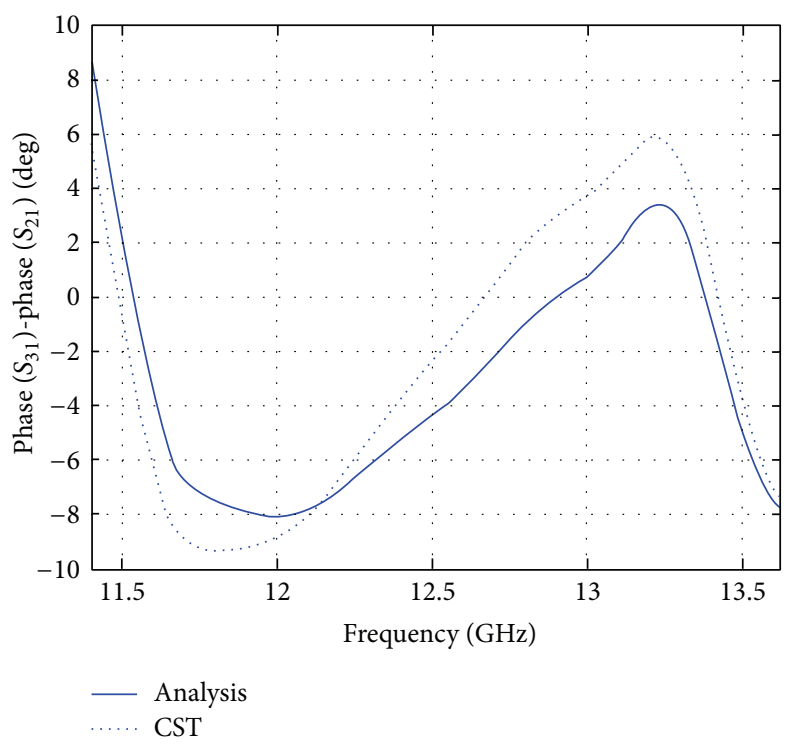

(a)

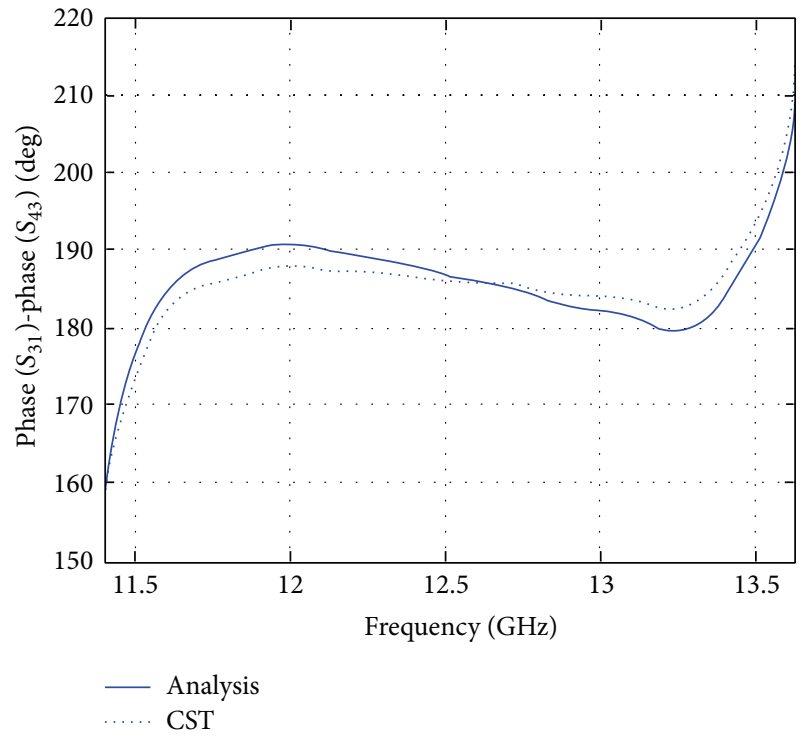

(b)

FIGURE 4: Phase responses of the SIW rat-race coupler: (a) in-phase response and (b) out-of-phase response.

By using (13),

$$
E_{2}(r)=\sum_{l=1}^{P_{\text {via }}} \sum_{m=1}^{N_{z}} \sum_{n=1}^{N_{\varphi}}\left(C_{l, m, n}^{\mathrm{TM}} Q_{n}+D_{l, m, n}^{\mathrm{TE}} P_{n}\right)
$$

To determine unknown coefficients $\left(C_{l, m, n}^{\mathrm{TM}}, D_{l, m, n}^{\mathrm{TE}}\right)$, the boundary conditions for the surface of each metallic should be imposed:

$$
\widehat{n} \times\left(E_{1}+E_{2}\right)=0
$$

in surface of each PEC post with radius $a_{i}$.

After imposing boundary conditions that span over the metal posts of sidewalls, arms, inner circle, inner conductor of coaxial cables, and four matching impedance metallic holes which are demonstrated in Figure 1(a), we can obtain a matrix (for TE and TM modes separately) for implantation in Matlab code. By using (16) the unknown coefficient vector $F_{l, m, n}^{\mathrm{TM} / \mathrm{TE}}=$ $\left[C_{l, m, n}^{\mathrm{TM}}, D_{l, m, n}^{\mathrm{TE}}\right]$ can be expressed as

$$
\left[\begin{array}{c}
F_{1, m, 0}^{\mathrm{TM} / \mathrm{TE}} \\
\vdots \\
F_{l, m, n}^{\mathrm{TM} / \mathrm{TE}}
\end{array}\right]=[\mathbf{U}]^{-1}[\mathbf{S}]
$$

where $U$ is interaction matrix between the vias and $S$ is excitation vector.

In (17), for propagation modes, we use the direct inverse command in Matlab to obtain unknown coefficients, and, for nonpropagation modes, we use iteration methods because of the singularity.
The admittance formulation of four coaxial cables in Figure 1(a) is

$$
Y^{\left(p_{i}, p_{j}\right)}=-\frac{\int \mathbf{H}^{\mathbf{p}_{i}} \cdot \mathbf{M}_{\mathbf{p}_{j}}^{\prime}(\boldsymbol{\rho}) \mathbf{d} \mathbf{S}^{\prime}}{|V|^{2}},
$$

where $\mathbf{M}^{\prime}(\boldsymbol{\rho})$ is surface current and has the same distribution as the $E$ field in a coaxial cable:

$$
\mathbf{E}^{\prime}(\boldsymbol{\rho})=\frac{V_{0}}{2 \rho \operatorname{Ln}(b / a)} \mathbf{a}_{\rho},
$$

and with Schelkunoff's equivalence principle:

$$
\mathbf{M}^{\prime}(\rho)=-2 \widehat{n} \times \mathbf{E}(\rho) .
$$

With excitation of the coupler with coaxial cables, the scattering parameters of the coupler conveniently can be obtained from (18).

\section{Optimization of the Rat-Race Coupler with IWO Algorithm}

Invasive weed optimization (IWO) algorithm has been first used by Mehrabian and Lucas [28] in dynamic and control systems theory. The main parameters involved in improving the bandwidth of a SIW hybrid ring coupler with IWO are $R 1$, $R 2$, and $R 3$. IWO parameters which are used for optimization are shown in Table 1 . In our optimization, we consider a goal function (goal: $\operatorname{Max}\left(S_{11}\right) \leq-15 \mathrm{~dB}$ ), and when we get to our goal after passing the necessary iterations, the other output results of the coupler will be satisfied. $S_{11}$ variations of the hybrid ring coupler in interest band versus number of iterations are shown in Figure 2. As shown in this figure, the optimized value of $S_{11}$ is $-15 \mathrm{~dB}$ after 175 iterations number which corresponds to the $R 1=4.5 \mathrm{~mm}, R 2=6.5 \mathrm{~mm}$, and $R 3=$ $15.5 \mathrm{~mm}$ over the $11.4-13.7 \mathrm{GHz}$ bandwidth. 

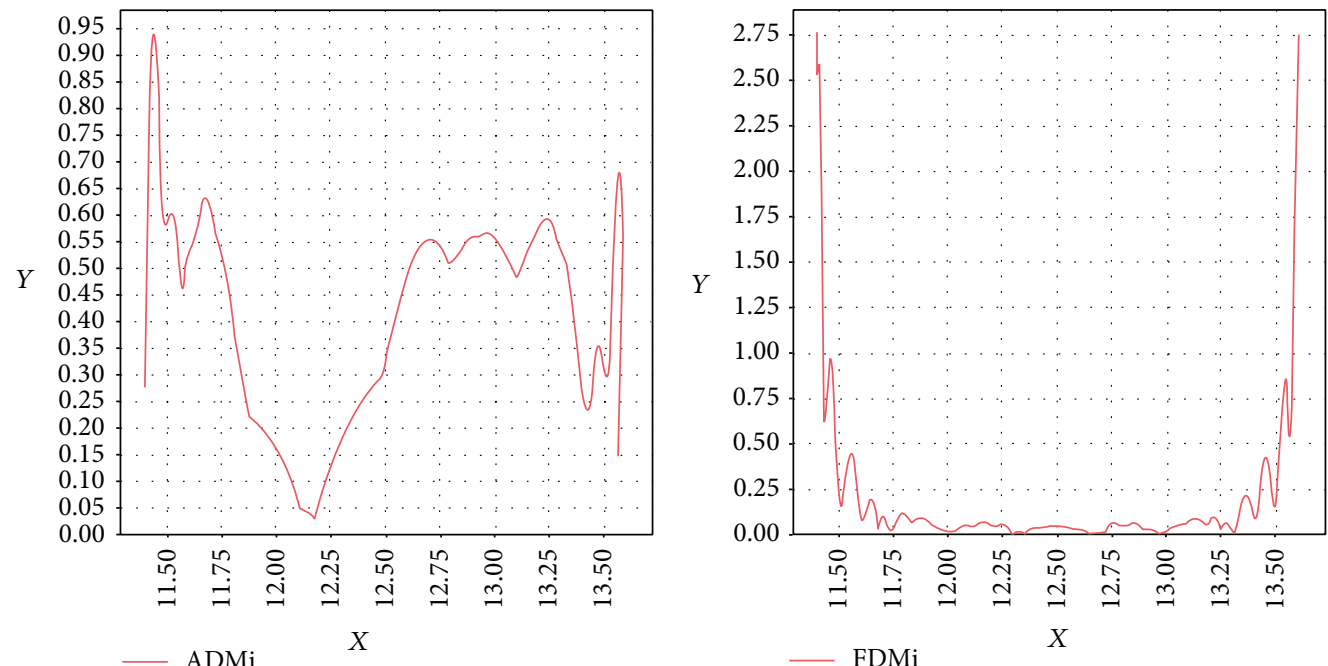

(a)
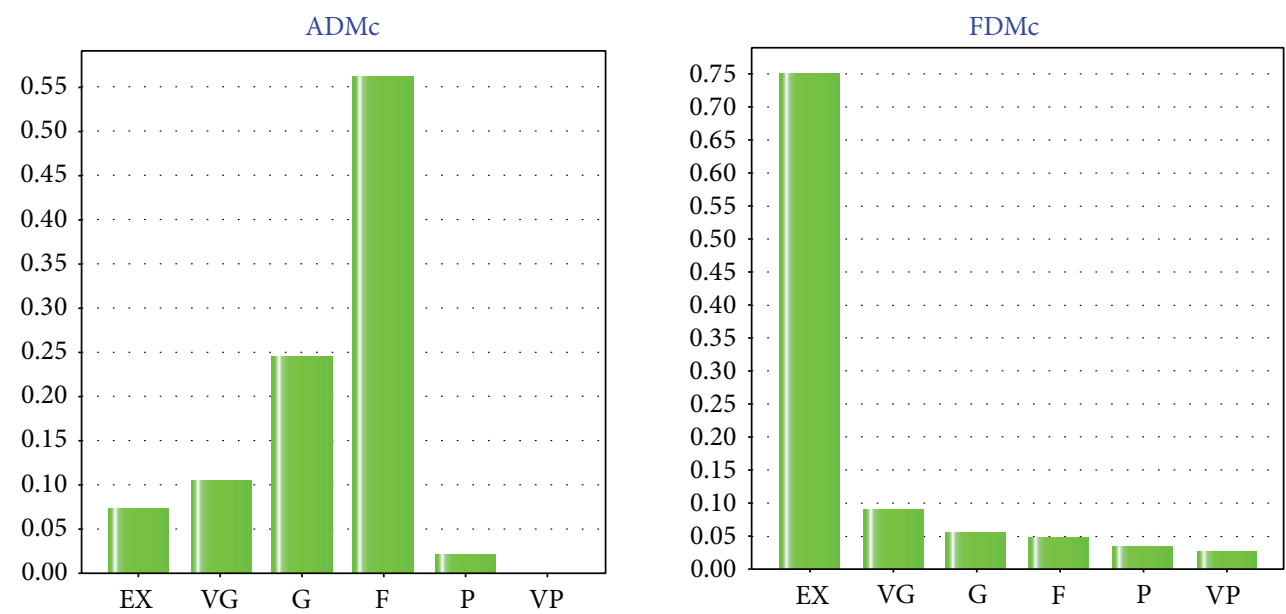

(b)
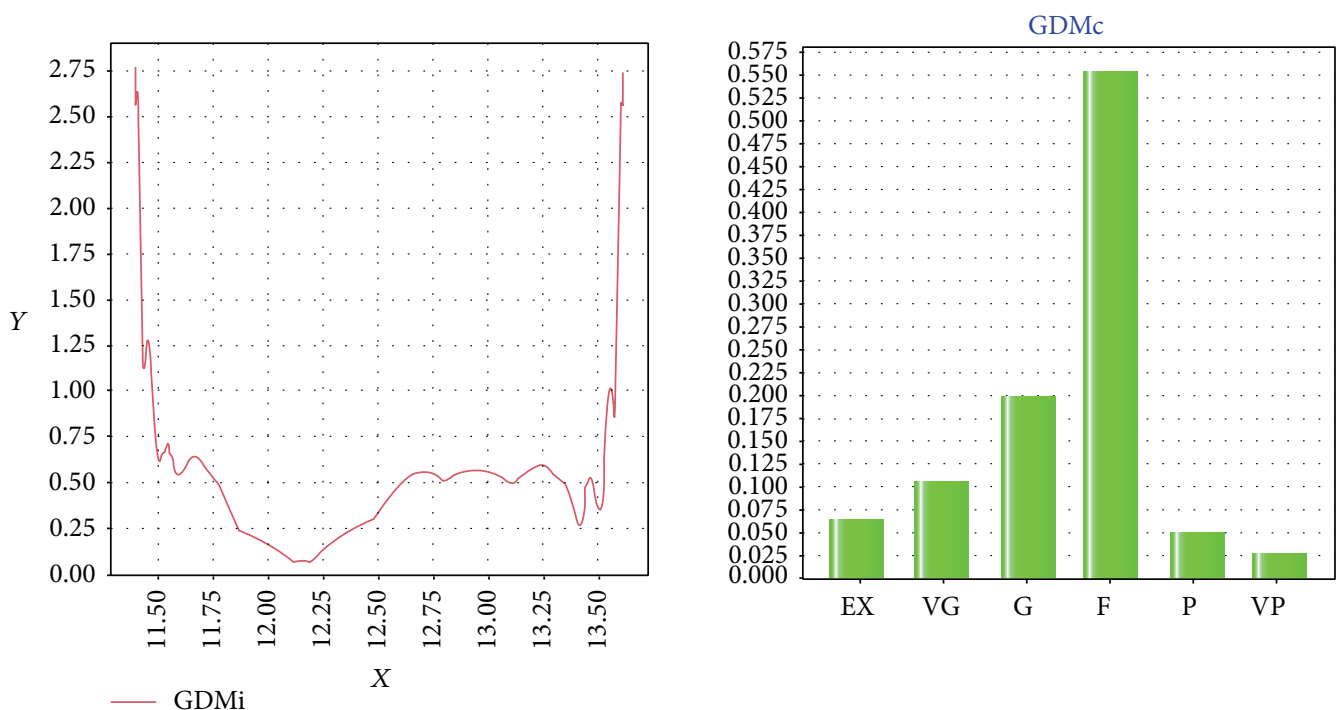

(c)

FIgUre 5: Continued. 


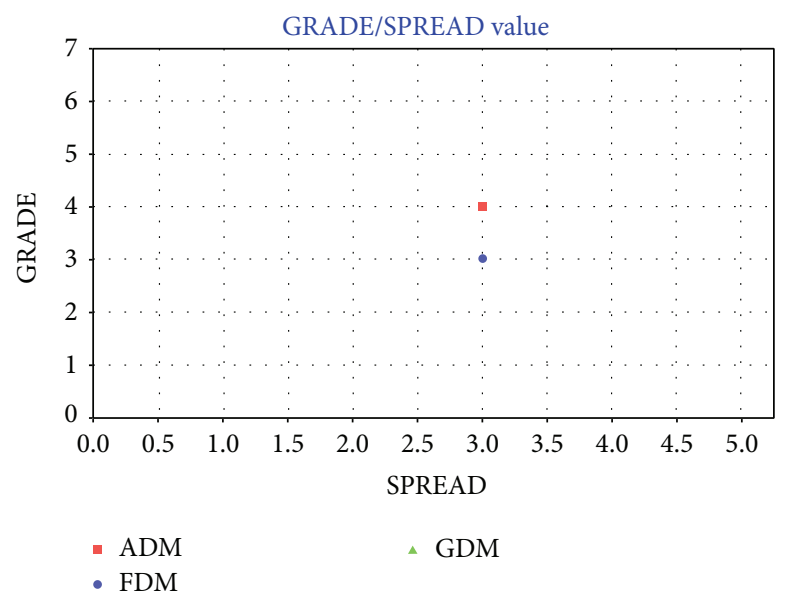

(d)

FIGURE 5: Agreement cases for in-phase responses with 1D FSV tool: (a) ADMi and FDMi, (b) ADMc and FDMc, (c) GDMi and GDMc, and (d) GRADE/SPREAD chart.

TABLE 1: IWO parameters values for Figure 1(a).

\begin{tabular}{lcc}
\hline Symbol & Quantity & Value \\
\hline No & Number of initial population & 5 \\
it $_{\max }$ & Maximum number of iterations & 1000 \\
$\operatorname{dim}$ & Problem dimension & 3 \\
$P_{\max }$ & Maximum number of plant population & 5 \\
$S_{\max }$ & Maximum number of seeds & 25 \\
$S_{\min }$ & Minimum number of seeds & 0 \\
$n$ & Nonlinear modulation index & 3 \\
$\sigma_{\text {initial }}$ & Initial value of standard deviation & 2 \\
$\sigma_{\text {final }}$ & Final value of standard deviation & 0.01 \\
$R 1_{\text {ini }}$ & & $2 \mathrm{~mm}$ to $20 \mathrm{~mm}$ \\
$R 2_{\text {ini }}$ & Initial search area & $4 \mathrm{~mm}$ to $30 \mathrm{~mm}$ \\
$R 3_{\text {ini }}$ & & $10 \mathrm{~mm}$ to $45 \mathrm{~mm}$ \\
\hline
\end{tabular}

\section{Feature Selective Validation (FSV)}

Feature selective validation (FSV) is a very useful technique to compare the agreement of two plots usually to decide that the two plots have excellent, very good, good, fair, poor, or very poor agreement [29]. By using FSV, the comparison between results is to be measured objectively, eliminating the element of subjectivity from the decision making process.

The FSV technique is based on the decomposition of the results to be compared into two component measures and then the recombination of the results to provide a global goodness of fit measure. The components used are three figures of merit of the comparison of two data sets [30-33]:

(1) the amplitude difference measure (ADM) that compares the amplitudes and trends of the two data sets,

(2) the feature difference measure (FDM) that compares the rapidly changing features (as a function of the independent variable),
(3) the global difference measure (GDM) that is obtained with combination of the ADM and FDM.

Accordingly, the comparison of two data sets can be ranked. This ranking is useful for making a selection between multiple comparisons. So, two quality factors for each figure of merit (ADM, FDM, and GDM) are regarded (i.e., the GRADE and the SPREAD).

The GRADE provides a numerical indication of the quality of the comparison and the SPREAD gives a numerical indication of the level of confidence that can be placed on this evaluation. In GRADE, the smaller it is, the better the comparison of the results is, and, in SPREAD, the higher the reliability is, the smaller it is. Furthermore, GRADE and SPREAD can be computed for each figure of merit and reported on a GRADE/SPREAD chart.

When we use FSV tool [34], there are time domain and frequency domain analyses. A time domain analysis is performed on two sets of data that are generic amplitude values.

In the case of frequency domain, there are three options to analyze:

(1) magnitude option that the input data are magnitude values,

(2) phase option that the input data are phase values,

(3) combined option in which the 1D FSV combined analysis performs two FSV analyses (i.e., magnitude and phase) and combines them in a single result.

\section{Result and Discussion}

The formulations in Section 2 and IWO algorithm have been implemented in Matlab code. The $S$-parameters from analysis, after optimization of the structure in Section 3, are shown in Figure 3. The $S_{11}$ (return loss) and $S_{14}$ (isolation) over 11.4-13.7 GHz are less than $-15 \mathrm{~dB}$ and $-18 \mathrm{~dB}$ in the order mentioned and insertion loss is divided equally $-3 \pm 0.2 \mathrm{~dB}$ in 

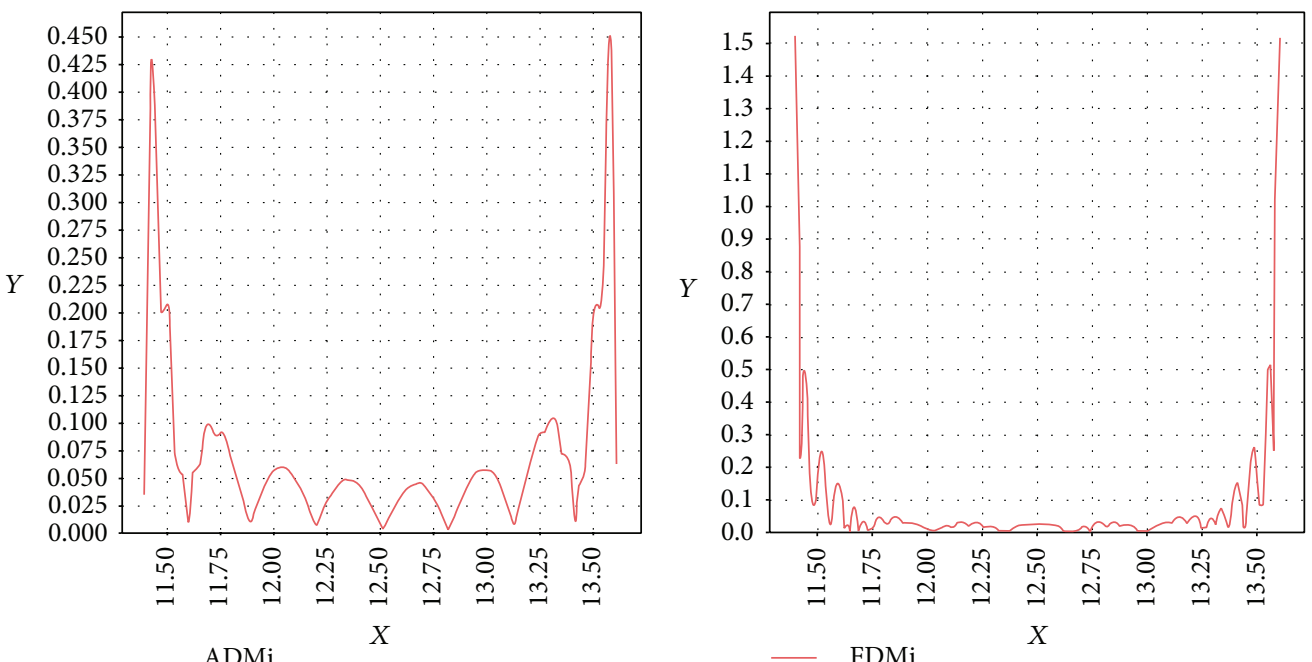

(a)
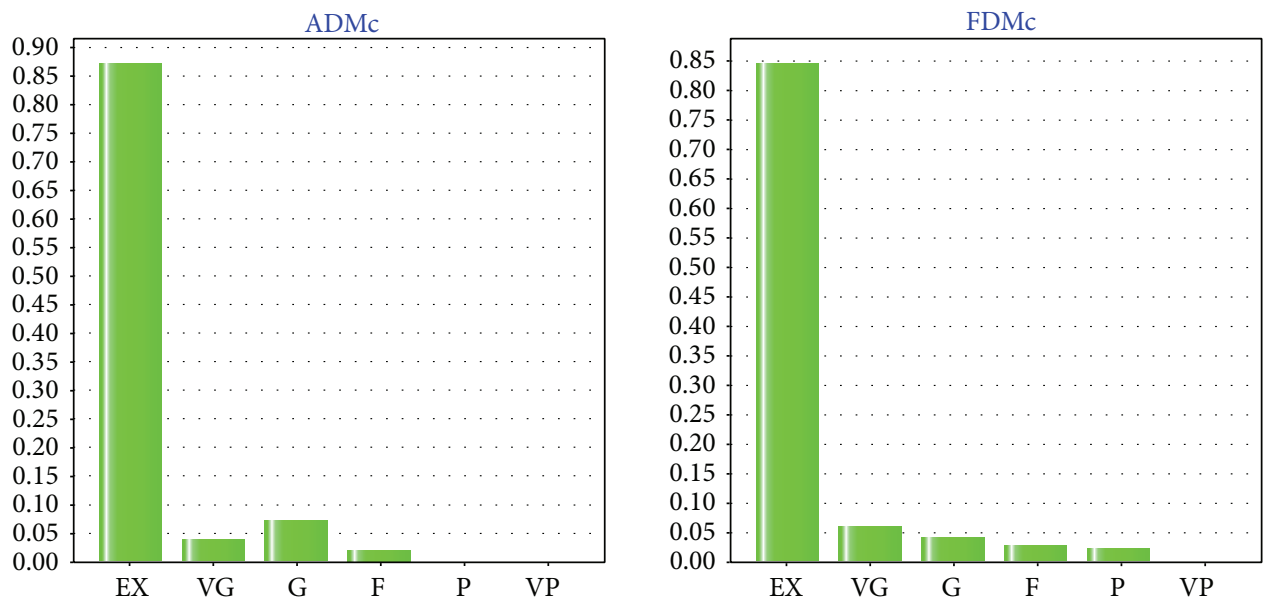

(b)
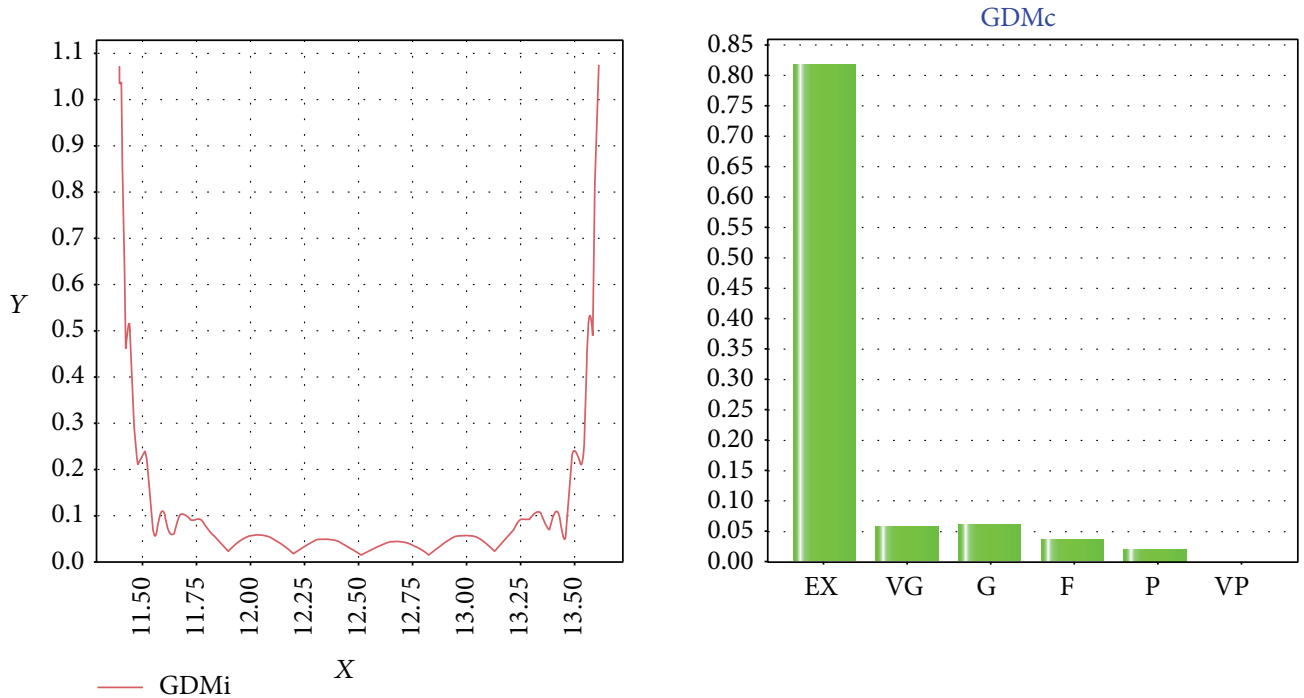

(c)

Figure 6: Continued. 


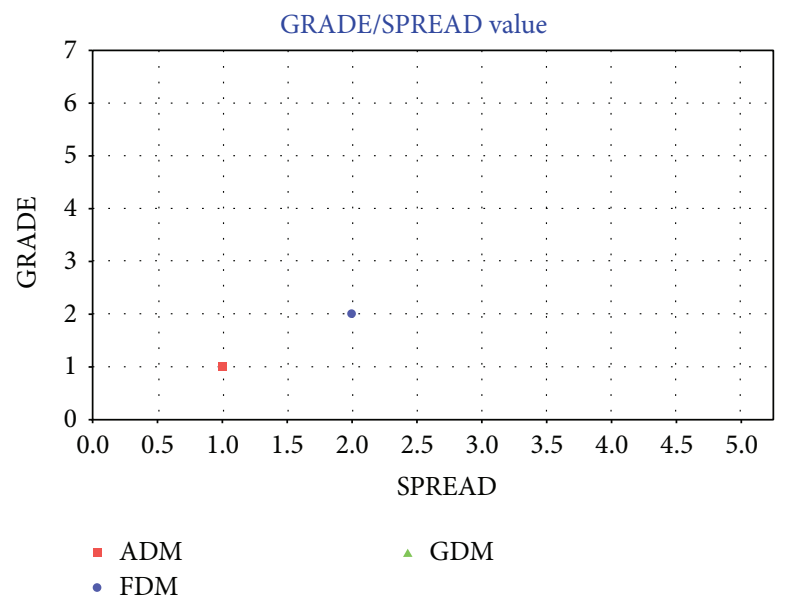

(d)

FIGURE 6: Agreement cases for out-of-phase responses with 1D FSV tool: (a) ADMi and FDMi, (b) ADMc and FDMc, (c) GDMi and GDMc, and (d) GRADE/SPREAD chart.

TABLE 2: Simulation time on a Core i5 with 4 GB RAM (azimuthally mode numbers $(n)=7$; vertically mode numbers $(m)=2$ ).

\begin{tabular}{lccc}
\hline Structure type in Figure 1 & Number of metallic vias & $\begin{array}{c}\text { CST CPU time } \\
\text { Time domain }\end{array}$ & $\begin{array}{r}\text { Analysis in this paper } \\
50 \text { frequency points }\end{array}$ \\
\hline Hybrid ring (rat-race) coupler & 330 & $1200 \mathrm{~s}$ & $110 \mathrm{~s}$ \\
\hline
\end{tabular}

ports 2 and 3. The analysis results show excellent agreement with the simulation. Figure 4 shows relative phases for the rat-race coupler. The phase difference in Figure 4(a) is $0 \pm 5^{\circ}$ and in Figure 4(b) is $180 \pm 10^{\circ}$ over the interest band. Also, there is good agreement between the two proposed methods. Obviously, the relative bandwidth after optimization from Figures 3 and 4 is approximately $17 \%$.

As an application of the FSV technique which has been explained in Section 4, the agreement of the output phase results in Figure 4 has been performed with 1D FSV tool 2.0.4 [32] in frequency domain. According to [34], excellent, very good, good, fair, poor, and very poor agreements fall in the ranges of [ $\left.\begin{array}{ll}0 & 0.1\end{array}\right),\left[\begin{array}{ll}0.1 & 0.2\end{array}\right),\left[\begin{array}{ll}0.2 & 0.4\end{array}\right),\left[\begin{array}{ll}0.4 & 0.8\end{array}\right),\left[\begin{array}{ll}0.8 & 1.6\end{array}\right)$, and [1.6 infinity), respectively. From Figure 5, it is observed that the FSV tool results show good and fair agreements for in-phase responses in the most range of the bandwidth of interest. Also, based on Figure 6, very good and excellent agreements have been obtained for out-of-phase responses in almost all of the operating frequency range.

To prove the efficiency of our analysis, simulation time for the considered case and comparing it to CST software are reported in Table 2. From this table, the run time ratio of the analysis related to CST is $1: 10.9$.

\section{Conclusion}

In this paper, the dyadic Green's function and vector wave functions for cylindrical coordination have been used for a SIW rat-race coupler. Our output results with the mode matching method after optimization with IWO and comparing it with CST using FSV technique and CPU time show accuracy and efficiency of our theory, respectively. Throughout $11.4-13.7 \mathrm{GHz}$, the structure has $17 \%$ relative bandwidth. By using the proposed analysis and optimizing the dimensions of the coupler, the bandwidth can shift in the interested band.

\section{Conflict of Interests}

The authors declare that there is no conflict of interests regarding the publication of this paper.

\section{References}

[1] K. Wu, D. Deslandes, and Y. Cassivi, “The substrate integrated circuits-a new concept for high-frequency electronics and optoelectronics," in Proceedings of the 6th International Conference on Telecommunications in Modern Satellite, Cable and Broadcasting Service (TELSIKS '03), vol. 1, pp. 3-10, 2003.

[2] R. Dehdasht-Heydari and M. Naser-Moghadasi, "Introduction of a novel technique for the reduction of cross polarization of rectangular microstrip patch antenna with elliptical DGS," Journal of Electromagnetic Waves and Applications, vol. 22, no. 8-9, pp. 1214-1222, 2008.

[3] J. Lacik, "Circularly polarized SIW square ring-slot antenna for X-band applications," Microwave and Optical Technology Letters, vol. 54, no. 11, pp. 2590-2594, 2012.

[4] K. Deng, Z. Guo, C. Li, and W. Che, "A compact planar bandpass filter with wide out-of-band rejection implemented by substrate-integrated waveguide and complementary split-ring resonator," Microwave and Optical Technology Letters, vol. 53, no. 7, pp. 1483-1487, 2011. 
[5] F. T. Ladani and R. Sh. Jam Safian, "A new siw resonator excitation method and its equivalent circuit," Microwave and Optical Technology Letters, vol. 55, no. 5, pp. 1133-1138, 2013.

[6] L. Xu, W. Che, L. Geng, and D. Wang, "Double-layer substrate integrated waveguide structure with variable fractional bandwidth," Microwave and Optical Technology Letters, vol. 49, no. 6, pp. 1459-1463, 2007.

[7] Z. Li, M. S. Mahani, and R. Abhari, "Experiment of substrate integrated waveguide interconnect measurement for high speed data transmission application," Microwave and Optical Technology Letters, vol. 54, no. 2, pp. 401-405, 2012.

[8] Q. F. Wei, Z. F. Li, and H. G. Shen, "Dual-mode filters based on substrate integrated waveguide by multilayer LTCC technology," Microwave and Optical Technology Letters, vol. 50, no. 11, pp. 2788-2790, 2008.

[9] L. Y. Liu, X. H. Tang, and T. Wu, "SIW-based low phase-noise millimeter-wave planar dual-port voltage-controlled oscillator," Journal of Electromagnetic Waves and Applications, vol. 27, no. 8-9, pp. 1059-1069, 2012.

[10] K. Song, F. Zhang, F. Chen, and Y. Fan, "Wideband millimetrewave four-way spatial power combiner based on multilayer SIW," Journal of Electromagnetic Waves and Applications, vol. 27, no. 13, pp. 1715-1719, 2013.

[11] J. Hirokawa and M. Ando, "Single-layer feed waveguide consisting of posts for plane tem wave excitation in parallel plates," IEEE Transactions on Antennas and Propagation, vol. 46, no. 5, pp. 625-630, 1998.

[12] F. Alessandri, M. Mongiardo, and R. Sorrentino, "A technique for the fullwave automatic synthesis of waveguide components: application to fixed phase shifters," IEEE Transactions on Microwave Theory and Techniques, vol. 40, no. 7, pp. 1484-1495, 1992.

[13] Y. Cassivi, L. Perregrini, P. Arcioni, M. Bressan, K. Wu, and G. Conciauro, "Dispersion characteristics of substrate integrated rectangular waveguide," IEEE Microwave and Wireless Components Letters, vol. 12, no. 9, pp. 333-335, 2002.

[14] L. Yan, W. Hong, K. Wu, and T. J. Cui, "Investigations on the propagation of the substrate integrated waveguide based on the method of lines," IEE Proceedings: Microwaves, Antennas and Propagation, vol. 152, no. 1, pp. 35-42, 2005.

[15] T. J. Coenen, D. J. Bekers, J. L. Tauritzt, and F. E. Van Vliet, "Design of post-wall waveguides with metal and dielectric posts," in Proceedings of the 37th European Microwave Conference (EUMC '07), pp. 20-23, Munich, Germany, October 2007.

[16] E. Díaz Caballero, H. Esteban, Á. Belenguer, and V. Boria, "Efficient analysis of substrate integrated waveguide devices using hybrid mode matching between cylindrical and guided modes," IEEE Transactions on Microwave Theory and Techniques, vol. 60, no. 2, pp. 232-243, 2012.

[17] A. Belenguer, H. Esteban, E. Diaz, C. Bachiller, J. Cascón, and V. E. Boria, "Hybrid technique plus fast frequency sweep for the efficient and accurate analysis of substrate integrated waveguide devices," IEEE Transactions on Microwave Theory and Techniques, vol. 59, no. 3, pp. 552-560, 2011.

[18] H. Chen, Q. Li, L. Tsang, C. C. Huang, and V. Jandhyala, "Analysis of a large number of vias and differential signaling in multilayered structures," IEEE Transactions on Microwave Theory and Techniques, vol. 51, no. 3, pp. 818-829, 2003.

[19] E. Arnieri and G. Amendola, "Analysis of substrate integrated waveguide structures based on the parallel-plate waveguide Green's function," IEEE Transactions on Microwave Theory and Techniques, vol. 56, no. 7, pp. 1615-1623, 2008.
[20] B. Wu and L. Tsang, "Full-wave modeling of multiple vias using differential signaling and shared antipad in multilayered high speed vertical interconnects," Progress in Electromagnetics Research, vol. 97, pp. 129-139, 2009.

[21] M. Casaletti, R. Sauleau, and M. Ettorre, "Efficient analysis of metallic and dielectric posts in parallel-plate waveguide structures," IEEE Transactions on Microwave Theory and Techniques, vol. 60, no. 10, pp. 2979-2989, 2012.

[22] B. Wu and L. Tsang, "Signal integrity analysis of package and printed circuit board with multiple vias in substrate of layered dielectrics," IEEE Transactions on Advanced Packaging, vol. 33, no. 2, pp. 510-516, 2010.

[23] E. Arnieri and G. Amendola, "Method of moments analysis of slotted substrate integrated waveguide arrays," IEEE Transactions on Antennas and Propagation, vol. 59, no. 4, pp. 1148-1154, 2011.

[24] H. Zairi, H. Baudrand, A. Gharsallah, and A. H. Gharbi, "An efficient iterative method for analysis of a substrate integrated waveguide structures," Microwave and Optical Technology Letters, vol. 52, no. 1, pp. 45-48, 2010.

[25] W. Che, K. Deng, E. K. N. Yung, and K. Wu, "H-plane 3-dB hybrid ring of high isolation in substrate-integrated rectangular waveguide (SIRW)," Microwave and Optical Technology Letters, vol. 48, no. 3, pp. 502-505, 2006.

[26] C. T. Tai, Generalized Vector and Dyadic Analysis: Applied Mathematics in Field Theory, Series on Electromagnetic Waves, IEEE Press, Piscataway, NJ, USA, 1991.

[27] C. T. Tai, Dyadic Green's Function in Electromagnetic Theory, Series on Electromagnetic Waves, IEEE Press, Piscataway, NJ, USA, 1993.

[28] A. R. Mehrabian and C. Lucas, "A novel numerical optimization algorithm inspired from weed colonization," Ecological Informatics, vol. 1, no. 4, pp. 355-366, 2006.

[29] "Standard for validation of computational electromagnetics computer modeling and simulation-part land 2," IEEE Standard P1597, 2008.

[30] A. P. Duffy, A. J. M. Martin, A. Orlandi, G. Antonini, T. M. Benson, and M. S. Woolfson, "Feature Selective Validation (FSV) for validation of computational electromagnetics (CEM). Part 1: the FSV method," IEEE Transactions on Electromagnetic Compatibility, vol. 48, no. 3, pp. 449-458, 2006.

[31] A. Orlandi, A. P. Duffy, B. Archambeault, G. Antonini, D. E. Coleby, and S. Connor, "Feature Selective Validation (FSV) for validation of computational electromagnetics (CEM). Part II: assessment of FSV performance," IEEE Transactions on Electromagnetic Compatibility, vol. 48, no. 3, pp. 460-467, 2006.

[32] G. Antonini, A. Ciccomancini Scogna, A. Orlandi, C. Ritota, and A. Duffy, "Applications of FSV to EMC and SI data," in Proceedings of the 2005 International Symposium on Electromagnetic Compatibility (EMC '05), pp. 278-283, Chicago, Ill, USA, August 2005.

[33] A. Duffy and A. Orlandi, "The influence of data density on the consistency of performance of the feature Selective Validation (FSV) technique," Applied Computational Electromagnetics Society Journal, vol. 21, no. 2, pp. 164-171, 2006.

[34] http://orlandi.ing.univaq.it/pub/FSV_Tool_20/. 

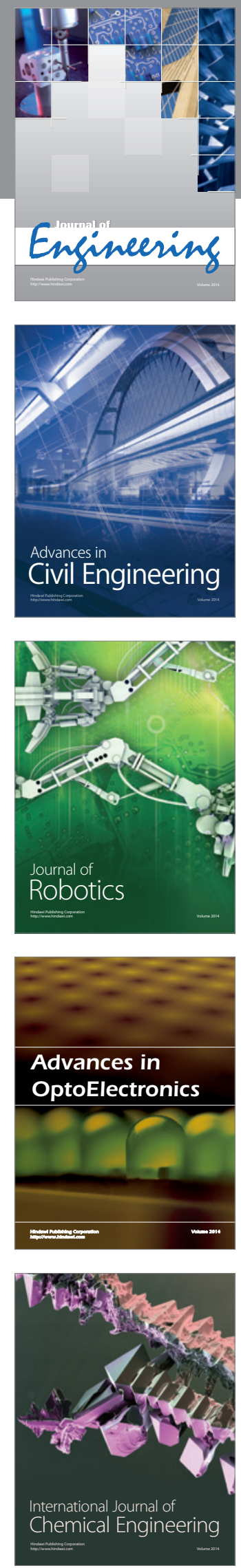

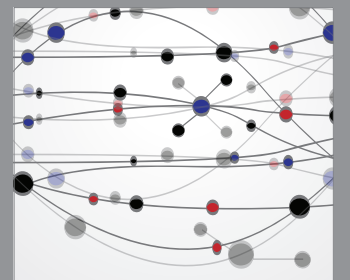

The Scientific World Journal
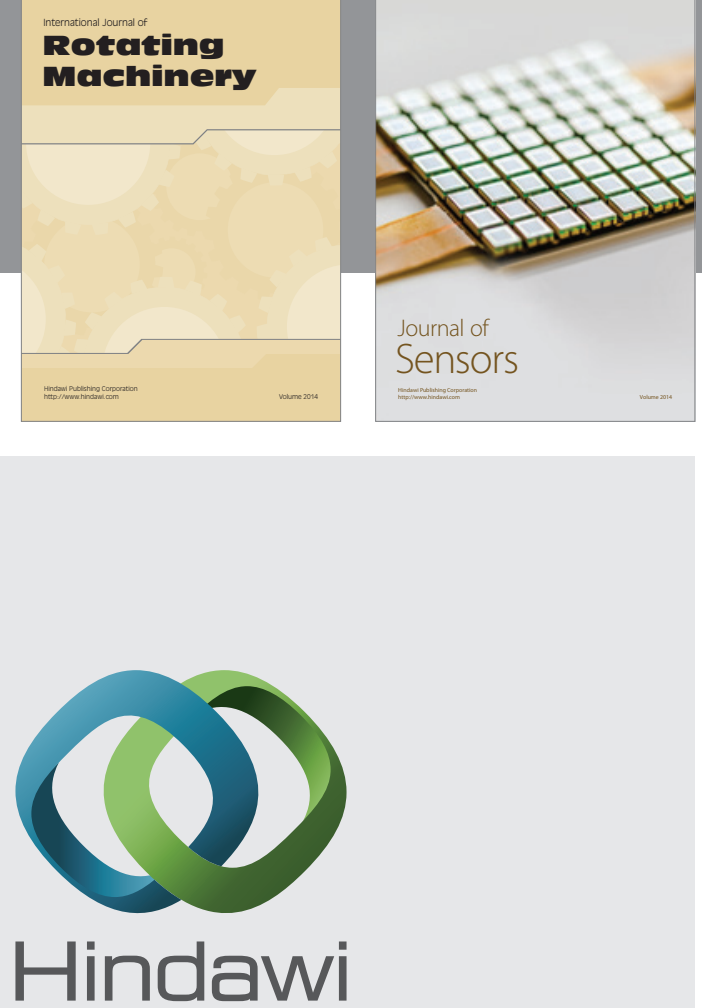

Submit your manuscripts at http://www.hindawi.com
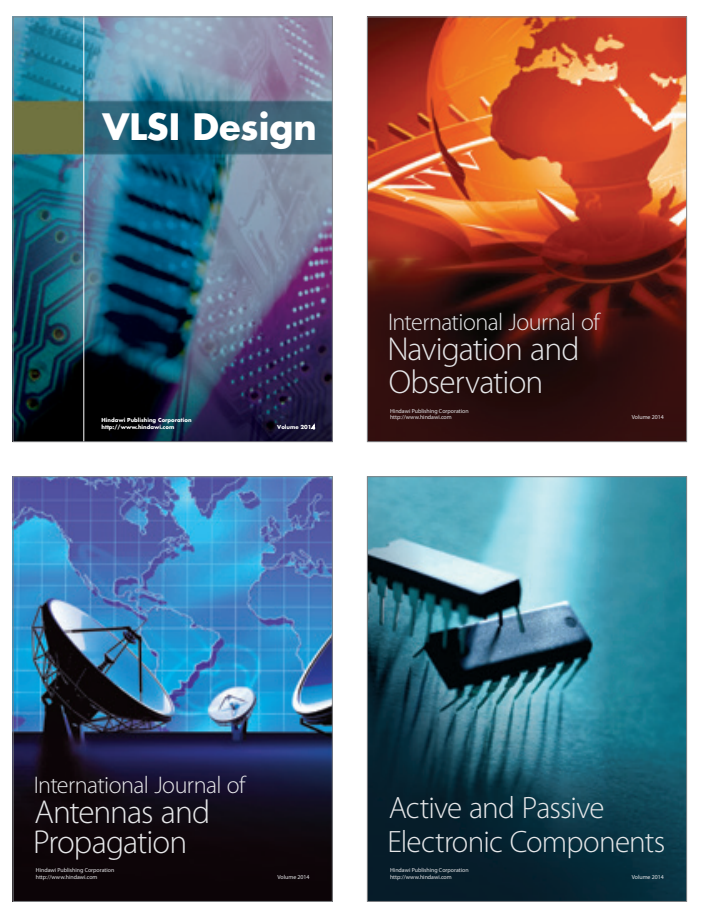
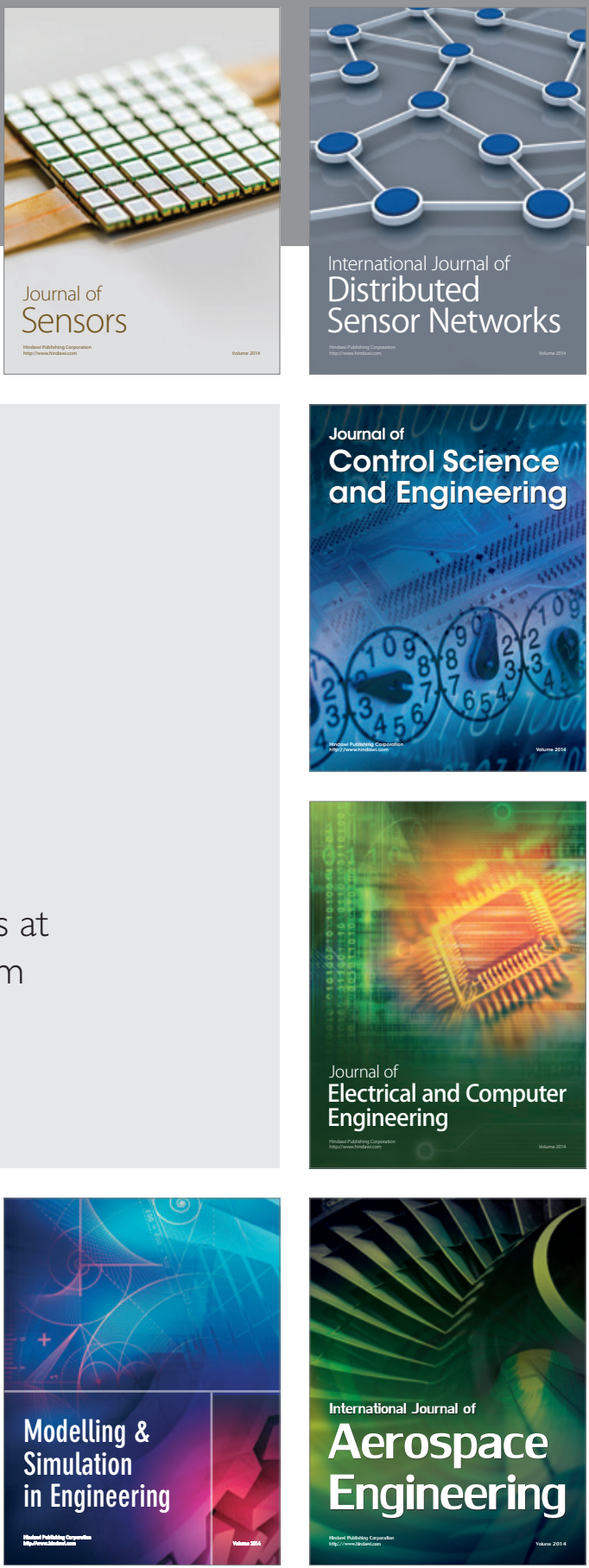

Journal of

Control Science

and Engineering
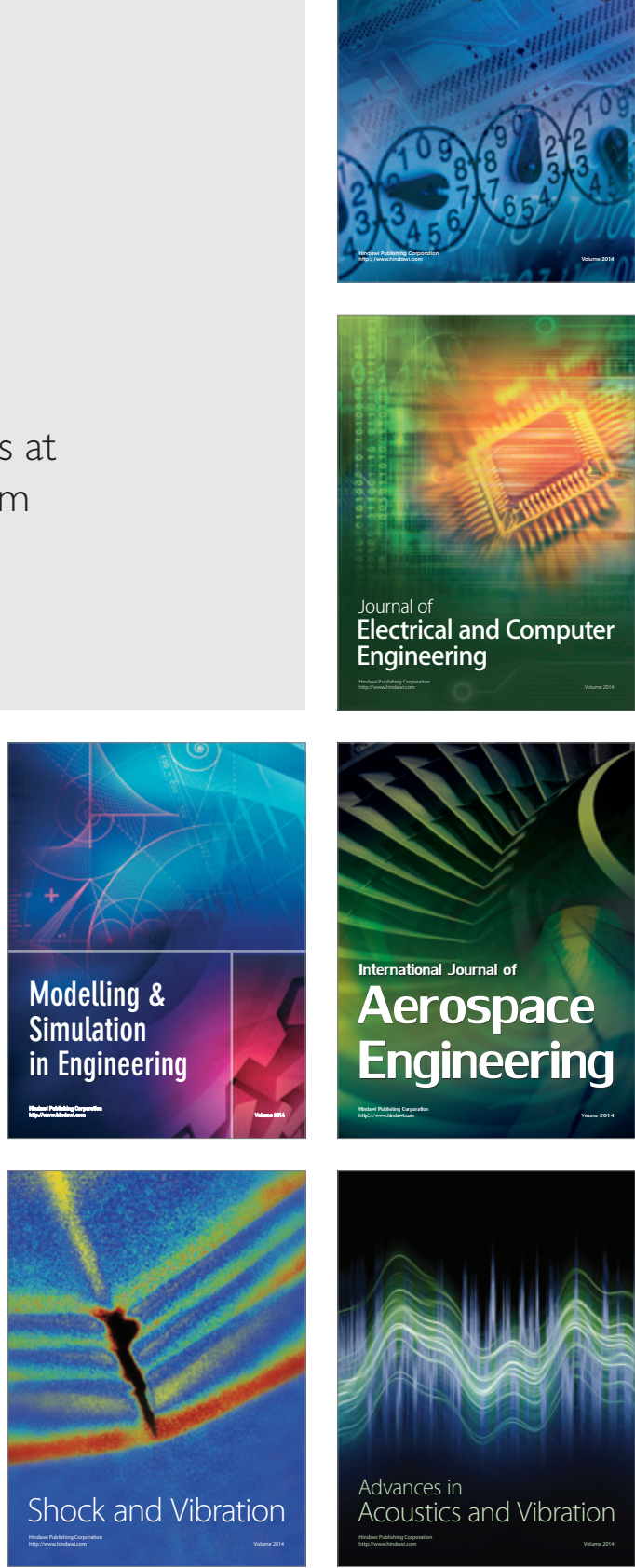